\title{
Ultrafast X-Ray Scattering of Xenon Nanoparticles: Imaging Transient States of Matter
}

\author{
C. Bostedt, ${ }^{1,2, *}$ E. Eremina, ${ }^{1}$ D. Rupp,${ }^{1}$ M. Adolph, ${ }^{1}$ H. Thomas,,${ }^{1}$ M. Hoener, ${ }^{1}$ A. R. B. de Castro, ${ }^{3}$ J. Tiggesbäumker, ${ }^{4}$ \\ K.-H. Meiwes-Broer, ${ }^{4}$ T. Laarmann, ${ }^{5}$ H. Wabnitz, ${ }^{5}$ E. Plönjes, ${ }^{5}$ R. Treusch, ${ }^{5}$ J. R. Schneider, ${ }^{5}$ and T. Möller ${ }^{1}$ \\ ${ }^{1}$ Institut für Optik und Atomare Physik, Technische Universität Berlin, Eugene-Wigner-Building EW 3-1, \\ Hardenbergstrasse 36, 10623 Berlin, Germany \\ ${ }^{2}$ SLAC National Accelerator Laboratory, P.O. Box 20450, Stanford, California 94309, USA \\ ${ }^{3}$ Laboratorio Nacional de Luz. Sincrotron, 13084-971 Campinas SP, Brazil and Instituto de Fisica Gleb Wataghin, \\ Universidade Estadual de Campinas, 13083-970 Campinas SP, Brazil \\ ${ }^{4}$ Institut für Physik, Universität Rostock, 18051 Rostock, Germany \\ ${ }^{5}$ Deutsches Elektronen-Synchrotron DESY, 22603 Hamburg, Germany
}

(Received 3 April 2011; published 29 February 2012)

\begin{abstract}
Femtosecond x-ray laser flashes with power densities of up to $10^{14} \mathrm{~W} / \mathrm{cm}^{2}$ at $13.7 \mathrm{~nm}$ wavelength were scattered by single xenon clusters in the gas phase. Similar to light scattering from atmospheric microparticles, the x-ray diffraction patterns carry information about the optical constants of the objects. However, the high flux of the x-ray laser induces severe transient changes of the electronic configuration, resulting in a tenfold increase of absorption in the developing nanoplasma. The modification in opaqueness can be correlated to strong atomic charging of the particle leading to excitation of $\mathrm{Xe}^{4+}$. It is shown that single-shot single-particle scattering on femtosecond time scales yields insight into ultrafast processes in highly excited systems where conventional spectroscopy techniques are inherently blind.
\end{abstract}

Ultrafast x-ray scattering opens the door for unprecedented insight into the structure and dynamics of matter with atomic resolution [1]. Any sample in the focus of an intense x-ray laser flash, however, will be converted into a highly excited, nonequilibrium plasma during the pulse [2-4]. The scattering signal is affected by such changes in the electronic structure of the sample, leading to modifications of the scattered intensities with respect to the expectations for the ground-state configuration [5]. The information about the electronic structure carried by the scattering pattern can be exploited to gain insight into transient electronic states on the femtosecond time scale.

In this Letter, we report on power-density-dependent single-shot single-particle scattering experiments on xenon clusters to investigate the interplay between excitation and scattering in nanoscale objects with x-ray pulses. Because of their finite size and simple electronic structure, atomic clusters are ideal objects for investigating the interaction between intense light pulses and matter in a wide spectral range from the infrared to $x$ rays [6]. For this study, a photon energy of $90.5 \mathrm{eV}$ was chosen. Xenon atoms and ions exhibit a greatly varying absorption cross section at $90.5 \mathrm{eV}$, including a strong absorption band for the $\mathrm{Xe}^{4+}$ ion with a cross section of up to $\sigma=200$ Mbarn [7,8]. Previous spectroscopy [9] and theoretical [10] investigations have shown that xenon is highly ionized under conditions similar to the current study. Ion spectra of xenon clusters irradiated under the same conditions, however, are dominated by singly charged monomer fragments due to efficient nanoplasma recombination [11], leaving questions about the ionization dynamics of nanometer-sized objects in intense short-wavelength laser pulses unanswered. The cluster expansion itself is inertia limited to subnanometer movements on the time scale of the pulse duration due to the high mass of xenon [12] so that the discussion of changes in the scattering patterns can be focused on electronic modifications in the xenon clusters.

The scattering data are modeled with Mie theory [13], yielding an analytical solution for light scattering of a sphere by solving the Maxwell equations for the incoming and outgoing (scattered) electromagnetic waves [14]. The variable parameters for the Mie fits are the particle size and the wavelength-dependent refractive index $n$. In the x-ray spectral regime, the refractive index is commonly expressed as $n=1-\delta+i \beta$. The real part $1-\delta$ is a measure of the phase shift of the scattered wave and connected to how efficiently the whole atom scatters compared to the Thomson scattering of a single free electron. The imaginary part $\beta$ describes the absorption of the system and is directly proportional to the photoabsorption cross section $\sigma$. In consequence, by measuring the scattering profiles and modeling with Mie theory, optical constants and thus information about the electronic structure of a particle can be determined [14].

The experiments have been performed at the FLASH free electron laser facility at DESY in Hamburg $[15,16]$. The experimental setup is shown in Fig. 1. The clusters are irradiated with ultrashort soft x-ray pulses $(\Delta t \sim 20 \mathrm{fs}, \lambda=$ $13.7 \pm 0.1 \mathrm{~nm}$ corresponding to $h \nu=90.5 \pm 0.7 \mathrm{eV})$ at power densities up to $(5 \pm 2) \times 10^{13} \mathrm{~W} / \mathrm{cm}^{2}$. The clusters are produced by expanding $\mathrm{Xe}$ in an Ar seed gas through a cooled, pulsed 200 micron diameter nozzle with an opening 
half angle of $4^{\circ}$ resulting in Xe cluster sizes ranging from $\sim 110-190 \mathrm{~nm}\left(\left(\langle N\rangle \sim 1-5 \times 10^{8}\right.\right.$ atoms $)$ and a median size of $\sim 140 \mathrm{~nm}\left(\langle N\rangle \sim 2 \times 10^{8}\right.$ atoms $)$ as deduced from the Mie fits. By skimming the cluster beam, it is ensured that, on average, less than one cluster is in the focal volume at a time. The scattering patterns are recorded with an imaging detector covering scattering angles from $\Theta=$ $3^{\circ}-50^{\circ}$ over a $2 \pi$ azimuth. For spectroscopy experiments, the imaging detector is replaced with an ion time-of-flight spectrometer, as reported previously [12], allowing us to detect ionic fragmentation products of the cluster. All data are stored shot-by-shot with a unique pulse identifier to correlate them to the laser parameters.

In Fig. 2, three representative single-shot single-particle scattering patterns out of the particle size distribution are shown for increasing power density of the incident $\mathrm{x}$-ray beam. The ring structure of the diffraction patterns reflects the almost spherical shape of the clusters similar to other single-particle imaging experiments [17]. Small intensity variations within the rings indicate that the particles are nanocrystals with surface facets [18] rather than perfect spherical droplets. In Fig. 2, an overproportional signal increase occurs for large scattering angles with increasing power densities, which have been deduced from the relative scaling of the Mie fits and the incoming pulse energies. As will be discussed in detail below, this effect cannot be described by a simple linear dependence on pulse energy or particle size.

To extract the scattering profiles, a slice of 20 degrees is cut out of the two-dimensional scattering pattern perpendicular to the polarization axis of the incoming light and

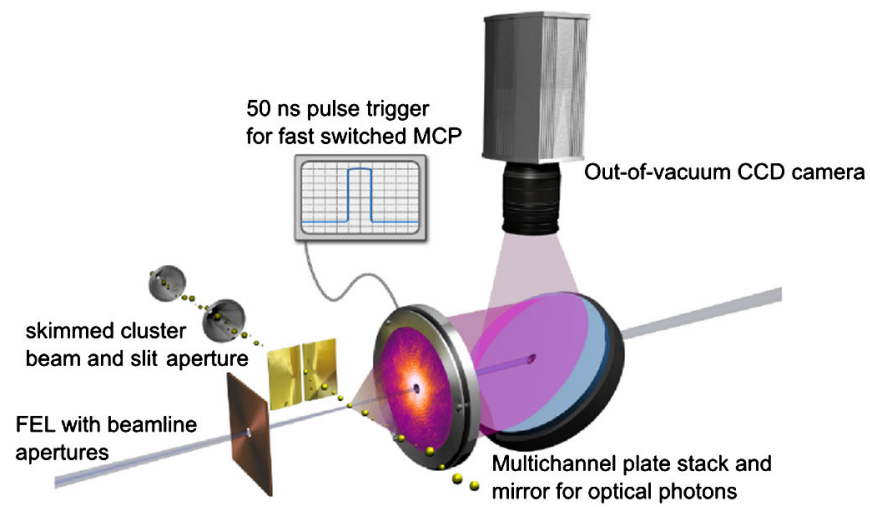

FIG. 1 (color online). Experimental setup for the single-shot nanoparticle imaging experiments. Nanoclusters are injected into the focused $\mathrm{x}$-ray pulse, and the scattered light is recorded with a high repetition rate detector. The x-ray photons are detected with an imaging quality multichannel plate (MCP) stack, converted into optical photons with a phosphorous screen, and digitized with an out-of-vacuum CCD camera. The multichannel plate stack is triggered to be active for only $50 \mathrm{~ns}$ during the arrival of the light pulse and then switched off prior to the arrival of any charged fragments from the explosion of the particle. averaged radially, i.e., over intensities at constant values of $q$, the momentum transfer of the photon during the scattering process. To correct for the flat detector, the intensities are multiplied with $\cos ^{-3} \Theta$. The resulting data are plotted in Fig. 3 over the dimensionless parameter $q R$ which is the product of the scattering vector $q=$ $4 \pi \lambda^{-1} \sin (\Theta / 2)\left[\mathrm{nm}^{-1}\right]$ and the particle radius $R[\mathrm{~nm}]$. The variable $q R$ reveals universal features of Mie scattering in the optical regime [19]. We adopt this concept which makes the intensity profiles of slightly different particle sizes comparable.

Figure 3(a) depicts a scattering profile from a Xe nanocluster taken with a power density of $2 \times 10^{13} \mathrm{~W} / \mathrm{cm}^{2}$. For
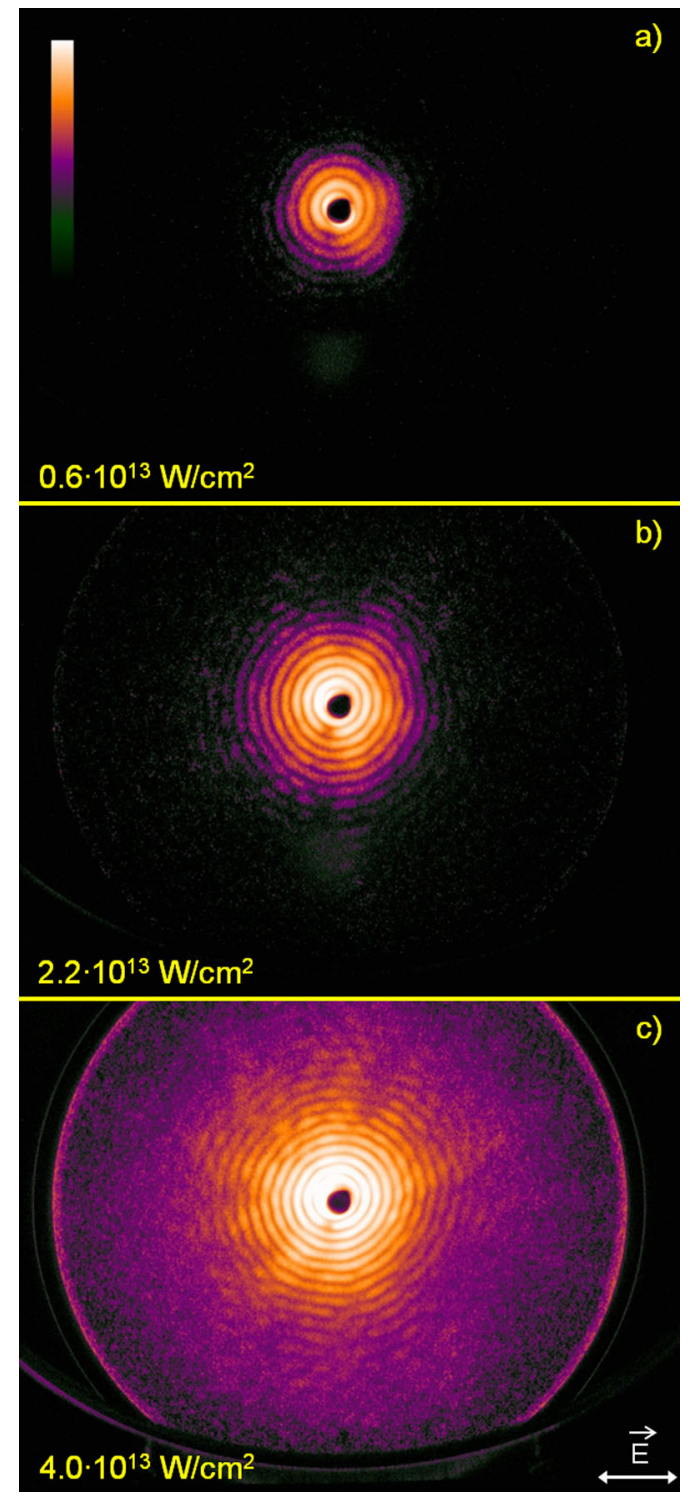

FIG. 2 (color online). Single-shot scattering patterns of similarly sized single clusters with increasing power density from top to bottom. For large scattering angles, the signal increases more than linearly with respect to the variation of the incoming power density. 

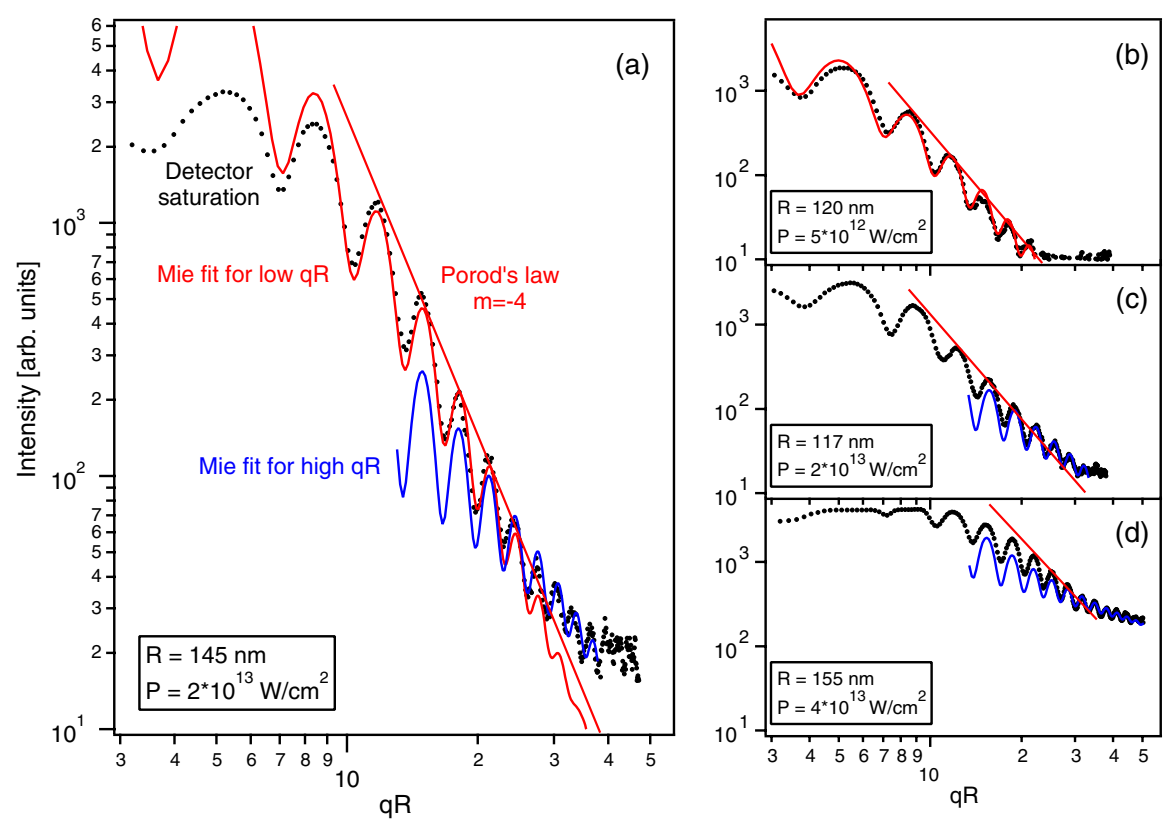

FIG. 3 (color online). Single-shot scattering profiles (dotted curves). In (a), the profile of a $R=145 \mathrm{~nm}$ cluster and two Mie fits for low and high $q R$ as well as Porod's law are shown. The three panels on the right-hand side correspond to the patterns shown in Fig. 2, demonstrating the power-density-dependent changes. The first maxima, in particular, for the highest power density shot in (d), suffer from detector saturation.

$q R>10$, the envelope of the maxima obeys a $(q R)^{-4}$ dependence known as Porod's law in small-angle x-ray scattering. Starting at the ninth maximum, i.e., roughly for $q R$ values $>25$, the envelope clearly deviates from the $(q R)^{-4}$ power law exhibiting a smaller slope. The corresponding length scale is $\sim 18 \mathrm{~nm}$, about 8 times smaller than the cluster radius of $\sim 150 \mathrm{~nm}$. Because of the obvious inadequacy of a purely geometric treatment, the following discussion focuses on possible contributions of the electronic structure to the scattering process.

To describe the exemplary data in Fig. 3(a), two independent Mie fits with the same particle radius are necessary. Generally, adjusting $\delta$ in the Mie fits predominantly alters the depth of the minima, while adjusting $\beta$ changes the slope of the envelope. The first fit in Fig. 3(a) for low $q R$ values [light gray (red) line] yields optical constants $\delta=0.04$ and $\beta=0.02$ which are in view of the error bars ( $\Delta \delta=0.04$ and $\Delta \beta=0.02$ to 0.05 depending on power density) in reasonable agreement with the neutral bulk xenon values of $\delta=-0.006$ and $\beta=0.04$. We like to note that the error bar of delta is quite large, since other effects, such as surface roughness, can effect the depth of the minima. Further, there is a zero-crossing of $\delta$ around $90 \mathrm{eV}$. The second fit, shown in dark gray (blue), describes the data in the tail for high $q R$. To fit the data for high $q R$, the absorption optical constant had to be adjusted to $\beta=$ 0.06 , while $\delta$ remained rather constant at 0.05 .

Figures 3(b)-3(d) show the laser power-densitydependent scattering profiles corresponding to the images in Fig. 2. For these profiles, it is apparent that the deviation from Porod's law becomes stronger with higher power densities. While the profile taken with $6 \times 10^{12} \mathrm{~W} / \mathrm{cm}^{2}$ [Fig. 3(b)] shows the $(q R)^{-4}$ dependence over the whole range, the profile taken with an order-of-magnitude higher power density [Fig. 3(d)] strongly deviates from the $(q R)^{-4}$ dependence, requiring strong adjustment of $\beta$ by more than an order of magnitude to 0.5 [dark gray (blue) curve], whereas $\delta$ again remains rather constant around 0.05 . For high power densities, the envelope slopes for the high $q R$ regime range from $(q R)^{-2.5}$ down to $(q R)^{-1}$. All observed changes are well within the linear detector response between $30-1000$ arbitrary units. It is interesting to note that the envelope slopes of the tail at high $q R$ show no dependence of the particle size. The data of a mid-sized particle in Fig. 3(a) and the smallest recorded particle in Fig. 3(c) taken with the same power densities yield similar optical constants for different sizes. Similarly, the largest recorded particle (not shown) with a radius of $190 \mathrm{~nm}$ and recorded with a power density of $1.7 \times 10^{13} \mathrm{~W} / \mathrm{cm}^{2}$ yields optical constants well on the trend line in the summary plot of Fig. 4.

The need of two independent sets of optical constants to describe the data is attributed to x-ray-induced electronic changes in the particle which alter its scattering response. The first fit [light gray (red) curve], similar for all power densities and in good agreement with Porod's law in smallangle x-ray scattering, describes the scattering for low power density pulses or at the beginning of a high power density pulse. The larger contribution for high $q R$, which becomes more dominant with higher power densities, is 


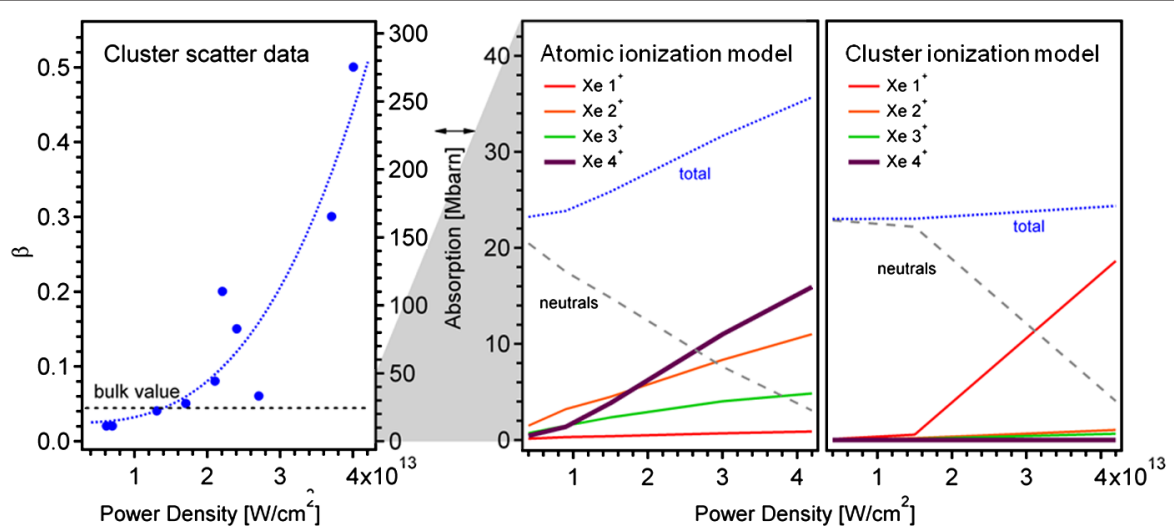

FIG. 4 (color online). Particle absorption parameter $\beta$ deduced from the scattering patterns. Right: Modeled relative absorption contribution, i.e., relative abundance times cross section, of the dominant ions based on atom and cluster target tof spectra recorded with similar laser conditions.

attributed to processes appearing at a later stage during the cluster-light interaction when the system has already been transformed to a plasma state. Therefore, the following discussion focuses on the power-density-dependent fraction of the signal appearing for high $q R$.

The extracted and strongly varying imaginary parts $\beta$ of the refractive index for high $q R$ values [dark gray (blue) curves in Fig. 3] as a function of power density are shown in the left panel of Fig. 4. At the lowest investigated power density of $6 \times 10^{12} \mathrm{~W} / \mathrm{cm}^{2}$, the absorption is comparable to the bulk value. For increasing power densities, the slopes of the envelopes of the high $q R$ fits start to decrease, requiring strong adjustment of $\beta$ in the Mie fits. At the highest investigated power density, the extracted $\beta$ exceeds the bulk value by more than a factor of 10 , corresponding to an absorption cross section exceeding 200 Mbarn.

The unexpectedly strong changes in the scattering pattern related to the increase in $\beta$ as a function of power density can be correlated to the ionization processes in the particle during the laser pulse. The scattering pattern is only generated during the pulse and therefore it is susceptible to the x-ray pulse-induced transient changes in the particle electronic structure. Vice versa, the scattering signal carries information about electronic structure changes during the light-matter interaction. To further discuss the impact of the individual charge states on the scatter process, each of their relative contributions in terms of their abundance times their respective cross section have to be estimated. For that purpose, two cluster ionization scenarios are developed: one is based on atomic ionization under similar conditions $[9,10]$ and one is based on cluster ion spectra recorded with the same setup and focusing conditions as the scattering patterns [12]. Ion time-of-flight (tof) spectroscopy shows that atomic xenon is efficiently ionized in the intense laser pulses at $90.5 \mathrm{eV}$ and that higher charge states rapidly become dominant in the spectra [9]. Specifically, the fraction of $\mathrm{Xe}^{4+}$ with its high absorption cross section reaching $200 \mathrm{Mbarn}$ is increasing by a factor of 100 over the observed power density window [10]. The cluster ion spectra recorded under similar conditions as the atomic data, however, look very different from the atomic spectra [12]. They are dominated by the singly charged monomer fragment due to efficient recombination of the created nanoplasma [11]. Because of these post-interaction effects, insight into the transient ionization dynamics of a nanometer-sized system has not yet been accessible with conventional spectroscopy techniques.

In the right two panels of Fig. 4, the solid lines show the measured relative abundance of the relevant charge states times their respective atomic cross section for atom $[9,10]$ and cluster [12] targets. The data based on the atom tof are dominated by contributions from higher charge states, and here, in particular, the $\mathrm{Xe}^{4+}$ signal becomes strongest for power densities beyond $2 \times 10^{13} \mathrm{~W} / \mathrm{cm}^{2}$ due to its large cross section. In contrast, the absorption data extracted from the cluster tof measurements show virtually exclusive contributions from singly charged monomer fragments due to nanoplasma recombination. For discussion of the total particle absorption, the contribution of neutral atoms has to be estimated because the scattering signal is sensitive to all atoms while tof spectroscopy only detects charged particles. In the first approximation, the neutral fraction is vanishing around $5 \times 10^{13} \mathrm{~W} / \mathrm{cm}^{2}$, where about one photon falls into each cross section $\sigma$, i.e., where all atoms should become ionized.

The development of the total absorption displayed in Fig. 4 shows a very different trend for the cluster and atom targets. In the cluster tof-based model, it basically stays flat with increasing power density, as the measured charge states have similar absorption cross sections to the neutrals of about 23 Mbarn. For the atom tof model, the total absorption increases with higher power density due to the growing abundance and large cross section of $\mathrm{Xe}^{4+}$. The cluster absorption parameter $\beta$ deduced from the scattering experiments is in its trend more comparable to the atom than the cluster tof data, indicating that the cluster is very 
efficiently ionized to higher charge states during the pulse and prior to recombination. The ionization deduced from the scatter patterns even exceeds the atomic effects, suggesting that it is even stronger during the pulse than in atom targets. Cluster-specific effects increasing the charge states on the cluster surface have been theoretically proposed [20] but could not yet experimentally be verified due to a lack of suitable and fast enough measurement techniques. Similarly, the plasma environment can lead to anomalous dispersion and a strong enhancement of absorption [21].

The $\mathrm{x}$-ray-induced changes to the electronic structure of the particle reflected in the strong increase of absorption as a function of power density affect the information carried by the scattering pattern, even though the particle geometry remains the same on the time scale of the interaction with the laser pulse. The smaller slope for high $q R$ values stems from scattering of a thin surface shell akin to a ring aperture, which results in a strong contribution of the particle surface to the scattering pattern.

In summary, the data show that single-shot singleparticle scattering yields insight into ultrafast processes in highly excited systems where conventional spectroscopy techniques such as tof mass spectroscopy are inherently blind. There, the particles are detected long after the lightmatter interaction took place and they are subject to secondary processes such as plasma recombination. Therefore, scattering experiments with intense ultrashort $\mathrm{x}$-ray pulses open new opportunities for investigation of transient electronic configurations of highly excited states of matter, nonequilibrium plasmas, and dense exotic systems, in addition to being key for solving structural information problems.

We would like to thank all staff at FLASH for their outstanding support. Funding is acknowledged from BMBF 05KS4KT1 and 05KS7KT2, as well as HGF Virtuelles Institut VH-VI-103 and VH-VI-302.

*Corresponding author. bostedt@slac.stanford.edu

[1] K. Gaffney and H. Chapman, Science 316, 1444 (2007).

[2] C. Bostedt, H. Thomas, M. Hoener, E. Eremina, T. Fennel, K.-H. Meiwes-Broer, H. Wabnitz, M. Kuhlmann, E. Plönjes, and K. Tiedtke et al., Phys. Rev. Lett. 100, 133401 (2008).
[3] H. Iwayama, K. Nagaya, M. Yao, H. Fukuzawa, X.-J. Liu, G. Prümper, M. Okunishi, K. Shimada, K. Ueda, and T. Harada et al., J. Phys. B 42, 134019 (2009).

[4] C. Bostedt, H. Thomas, M. Hoener, T. Möller, U. Saalmann, I. Georgescu, C. Gnodtke, and J.-M. Rost, New J. Phys. 12, 083004 (2010).

[5] S. P. Hau-Riege, Phys. Rev. A 76, 042511 (2007).

[6] C. Bostedt, M. Adolph, E. Eremina, M. Hoener, D. Rupp, S. Schorb, H. Thomas, A. R. B. de Castro, and T. Möller, J. Phys. B 43, 194011 (2010).

[7] P. Andersen, T. Andersen, F. Folkmann, V. Ivanov, H. Kjeldsen, and J. West, J. Phys. B 34, 2009 (2001).

[8] A. Aguilar, J. D. Gillaspy, G. F. Gribakin, R. A. Phaneuf, M. F. Gharaibeh, M. G. Kozlov, J. D. Bozek, and A. L. D. Kilcoyne, Phys. Rev. A 73, 032717 (2006).

[9] A. A. Sorokin, S. V. Bobashev, T. Feigl, K. Tiedtke, H. Wabnitz, and M. Richter, Phys. Rev. Lett. 99, 213002 (2007).

[10] M. G. Makris, P. Lambropoulos, and A. Mihelic, Phys. Rev. Lett. 102, 033002 (2009).

[11] M. Hoener, C. Bostedt, H. Thomas, L. Landt, E. Eremina, H. Wabnitz, T. Laarmann, R. Treusch, A. R. B. de Castro, and T. Möller, J. Phys. B 41, 181001 (2008).

[12] H. Thomas, C. Bostedt, M. Hoener, E. Eremina, H. Wabnitz, T. Laarmann, E. Ploenjes, R. Treusch, A. R. B. de Castro, and T. Möller, J. Phys. B 42, 134018 (2009).

[13] G. Mie, Ann. Phys. (Leipzig) 330, 377 (1908).

[14] C. Bohren and D. Huffman, Absorption and Scattering of Light by Small Particles (Wiley-VCH, New York, 1983).

[15] W. Ackermannet al., Nature Photon. 1, 336 (2007).

[16] K. Tiedtke, A. Azima, N. von Bargen, L. Bittner, S. Bonfigt, S. Duesterer, B. Faatz, U. Fruehling, M. Gensch, and C. Gerth et al., New J. Phys. 11, 023029 (2009).

[17] M. J. Bogan, W. H. Benner, S. Boutet, U. Rohner, M. Frank, A. Barty, M. M. Seibert, F. Maia, S. Marchesini, and S. Bajt et al., Nano Lett. 8, 310 (2008).

[18] C. G. Schroer, P. Boye, J. M. Feldkamp, J. Patommel, A. Schropp, A. Schwab, S. Stephan, M. Burghammer, S. Schöder, and C. Riekel, Phys. Rev. Lett. 101, 090801 (2008).

[19] C. Sorensen and D. Fischbach, Opt. Commun. 173, 145 (2000).

[20] C. Gnodtke, U. Saalmann, and J. M. Rost, Phys. Rev. A 79, 041201 (2009).

[21] J. Nilsen, W. Johnson, and K. Cheng, Proc. SPIE Int. Soc. Opt. Eng. 6702, 67020N (2007). 